TITLE:

\title{
Net quark number probability distribution near the chiral crossover transition
}

\section{$\operatorname{AUTHOR}(\mathrm{S})$ :}

Morita, Kenji; Friman, Bengt; Redlich, Krzysztof; Skokov, Vladimir

\section{CITATION:}

Morita, Kenji ... [et al]. Net quark number probability distribution near the chiral crossover transition. Physical Review C 2013, 88(3): 034903.

ISSUE DATE:

2013-09-12

URL:

http://hdl.handle.net/2433/179417

RIGHT:

(C)2013 American Physical Society 


\title{
Net quark number probability distribution near the chiral crossover transition
}

\author{
Kenji Morita, ${ }^{1,2, *}$ Bengt Friman, ${ }^{3}$ Krzysztof Redlich,${ }^{2,4}$ and Vladimir Skokov ${ }^{5, \dagger}$ \\ ${ }^{1}$ Yukawa Institute for Theoretical Physics, Kyoto University, Kyoto 606-8502, Japan \\ ${ }^{2}$ Extreme Matter Institute EMMI, GSI, Planckstrasse 1, D-64291 Darmstadt, Germany \\ ${ }^{3}$ GSI, Helmholzzentrum für Schwerionenforschung, Planckstrasse 1, D-64291 Darmstadt, Germany \\ ${ }^{4}$ Institute of Theoretical Physics, University of Wroclaw, PL-50204 Wroctaw, Poland \\ ${ }^{5}$ Physics Department, Brookhaven National Laboratory, Upton, New York 11973, USA
}

(Received 12 July 2013; published 12 September 2013)

\begin{abstract}
We investigate properties of the probability distribution of the net quark number near the chiral crossover transition in the quark-meson model. The calculations are performed within the functional renormalization group approach, as well as in the mean-field approximation. We find that there is a substantial influence of the underlying chiral phase transition on the properties of the probability distribution. In particular, for a physical pion mass, the distribution which includes the effect of mesonic fluctuations, differs considerably from both, the mean-field and Skellam distributions. The latter is considered as a reference for a noncritical behavior. A characteristic feature of the net quark number probability distribution is that, in the vicinity of the chiral crossover transition in the $O(4)$ universality class, it is narrower than the corresponding mean-field and Skellam function. We study the volume dependence of the probability distribution, as well as the resulting cumulants, and discuss their approximate scaling properties.
\end{abstract}

DOI: 10.1103/PhysRevC.88.034903

PACS number(s): 25.75.Nq, 25.75.Gz, 24.60.-k, 12.39.Fe

\section{INTRODUCTION}

The structure of the QCD phase diagram is one of the fundamental problems addressed in both theoretical and experimental studies [1,2]. At finite chemical potential, the existence of a critical point (CP) has been conjectured, based on effective chiral models [3] and preliminary lattice QCD results [4]. The fluctuations of the conserved changes have been proposed as a signature for the conjectured CP [5-10]. However, in this paper we focus on the QCD phase transition at small net baryon densities.

It was conjectured by Pisarski and Wilczek [11] that for massless light quarks, the QCD phase transition is of the second order, belonging to the $O(4)$ universality class. Current lattice QCD simulations at physical quark masses show, that at vanishing baryon density the transition from a hadron gas to quark matter is of the crossover type [12]. This implies that the corresponding singularity in the thermodynamic observables is shifted to complex values of the baryon chemical potential [13] and the experimental signatures of the phase transition could be washed out.

The conjecture of Pisarski and Wilczek is supported by recent lattice QCD studies [14,15], which show that for physical values of the light quark masses, the magnetic equation of state of QCD is consistent with the $O(4)$ scaling. This result has opened new opportunities to verify the QCD phase boundary experimentally by measuring fluctuations of conserved charges [5,16-20]. Indeed, based on the residual

\footnotetext{
*Present address: Frankfurt Institute for Advanced Studies, Ruth-Moufang-Str. 1, D-60438 Frankfurt am Main, Germany; kmorita@yukawa.kyoto-u.ac.jp.

${ }^{\dagger}$ Present address: Department of Physics, Western Michigan University, 1903 W. Michigan Avenue, Kalamazoo, MI 49008, USA.
}

$O$ (4) criticality and the proximity of the chiral crossover transition to the freeze-out line in heavy ion collisions, the characteristic modifications of the fluctuations of conserved charges have been proposed as a signature for the QCD phase boundary at small net baryon densities [5,8,16,21-23]. It has been shown, that at the chiral crossover transition, the higher order cumulants of the net baryon number and electric charge can be negative, owing to the $O(4)$ scaling $[22,23]$.

Such cumulants have recently been explored in heavy ion collisions by STAR Collaboration [24,25]. The data show deviations from the Skellam distribution, which are qualitatively consistent with theoretical expectations based on the $O(4)$ chiral critical dynamics. We note, however, that the role of uncertainties associated with the event-by-event measurements of fluctuations remain to be clarified [26-29].

Cumulants of net charges have also been studied in first principle lattice QCD calculations [19,20,30-32], as well as in effective chiral models [21-23,33-40]. Their properties are consistent with general expectations based on the $O(4)$ scaling free energy.

Fluctuations of conserved charges are directly linked to the corresponding probability distribution. Thus, the critical properties of cumulants of conserved charges must also be reflected in the probability distribution.

Recently, the effects of the chiral phase transition on the net baryon number probability distribution was examined within the framework of mean-field theory and the scaling theory of phase transitions [41]. It was found, that the critical behavior of the cumulants is a consequence of the change of the corresponding probability distribution.

In the present work, we extend our previous studies to a more realistic model. We consider the two-flavor quarkmeson model which is a low energy effective theory for the chiral properties of QCD. The critical fluctuations are treated 
consistently by means of the functional renormalization group (FRG) [42-44].

In the quark-meson model the coupling of quarks to the Polyakov loop, which is important for implementation of the statistical confinement properties, is neglected. However, within the FRG approach, the $O(4)$ scaling behavior and thermodynamics near the $\mathrm{CP}$ are well described by this model [45-48].

We show that there is a substantial influence of the underlying chiral phase transition on the properties of the probability distribution. In particular, we find that for a physical pion mass, the distribution which includes the effect of mesonic fluctuations, differs considerably from both, the mean-field and Skellam distributions. The latter is considered as a reference for a noncritical behavior. A characteristic feature of the net quark number probability distribution is that in the vicinity of a chiral crossover transition of the $O$ (4) universality class, it is narrower than the corresponding mean-field and Skellam distributions.

This paper is organized as follows. In the next section, we introduce the quark-meson model and its thermodynamic properties. In Sec. III, we present results on the probability distribution and different cumulants of the net quark number. Section IV is devoted to the concluding remarks.

\section{THE THERMODYNAMIC POTENTIAL IN THE QUARK-MESON MODEL}

We employ the quark-meson model to explore the influence of the chiral phase transition on the probability distribution of the net quark-number.

The quark-meson model is an effective realization of low energy QCD in which the $O(4)$ chiral meson multiplet $\phi=$ $(\sigma, \vec{\pi})$ is coupled to quark fields. The Lagrangian density is given by

$$
\begin{aligned}
\mathcal{L}= & \bar{q}\left[i \gamma_{\mu} \partial^{\mu}-g\left(\sigma+i \gamma_{5} \vec{\tau} \cdot \vec{\pi}\right)\right] q+\frac{1}{2}\left(\partial_{\mu} \sigma\right)^{2}+\frac{1}{2}\left(\partial_{\mu} \vec{\pi}\right)^{2} \\
& -U(\sigma, \vec{\pi}),
\end{aligned}
$$

where $U(\sigma)$ denotes the meson potential,

$$
U(\sigma, \vec{\pi})=\frac{1}{2} m^{2} \phi^{2}+\frac{\lambda}{4} \phi^{4}-h \sigma .
$$

For $m^{2}<0$ and $h=0$, the $O(4)$ symmetry of the potential is spontaneously broken to $O(3)$, resulting in a nonvanishing value of the vacuum scalar condensate $\langle\sigma\rangle$ and a nonzero quark mass. The last term, $h=f_{\pi} m_{\pi}^{2}$, breaks the chiral symmetry explicitly and yields the nonzero pion mass.

We obtain the thermodynamics of the quark-meson model by computing the thermodynamic potential within the FRG approach, as discussed in Ref. [45]. Following [42], we consider a scale dependent effective action in the local potential approximation. Thereby, we neglect the wave function renormalization and the flow of the Yukawa coupling. Using the so-called optimized cutoff functions, one obtains the evolution equation for the scale dependent thermodynamic potential density [45] with the reduced field variable

$$
\begin{aligned}
& \rho=\left(\sigma^{2}+\vec{\pi}^{2}\right) / 2 \\
& \partial_{k} \Omega_{k}(\rho)= \frac{k^{4}}{12 \pi^{2}}\left[\frac{3}{E_{\pi}}\left\{1+2 n_{B}\left(E_{\pi}\right)\right\}+\frac{1}{E_{\sigma}}\left\{1+2 n_{B}\left(E_{\sigma}\right)\right\}\right. \\
&\left.-\frac{2 v_{q}}{E_{q}}\left\{1-n_{F}\left(E_{q}^{+}\right)-n_{F}\left(E_{q}^{-}\right)\right\}\right]
\end{aligned}
$$

where $n_{B}$ and $n_{F}$ are the Bose and the Fermi distribution functions, respectively, and $v_{q}=2 N_{c} N_{f}=12$ is the quark degeneracy. The single particle energies of pion, $\sigma$ meson, and quark/antiquark are given by

$$
\begin{aligned}
& E_{\pi}=\sqrt{k^{2}+\bar{\Omega}_{k}^{\prime}}, \quad E_{\sigma}=\sqrt{k^{2}+\bar{\Omega}_{k}^{\prime}+2 \rho \bar{\Omega}_{k}^{\prime \prime}}, \\
& E_{q}^{ \pm}=\sqrt{k^{2}+2 g^{2} \rho} \pm \mu,
\end{aligned}
$$

where $\bar{\Omega}_{k}^{\prime}$ and $\bar{\Omega}_{k}^{\prime \prime}$ denote the first and the second derivatives of $\bar{\Omega}_{k}=\Omega_{k}+h \sqrt{2 \rho_{k}}$, with respect to $\rho$. The flow equation (3) is solved by using the Taylor expansion method. Expanding the potential up to the third order in $\rho$ around the potential minimum $\rho_{k}$,

$$
\Omega_{k}(\rho)=\sum_{n=0}^{3} \frac{a_{n}(k)}{n !}\left(\rho-\rho_{k}\right)^{n},
$$

and using Eq. (3), one finds the flow equations for the coefficients $a_{n}(k)$ and $\rho_{k}$,

$$
\begin{aligned}
d_{k} a_{0, k} & =\frac{c}{\sqrt{2 \rho_{k}}} d_{k} \rho_{k}+\partial_{k} \Omega_{k}, \\
d_{k} \rho_{k} & =-\frac{1}{\left(c /\left(2 \rho_{k}\right)^{3 / 2}+a_{2, k}\right)} \partial_{k} \Omega_{k}^{\prime}, \\
d_{k} a_{2, k} & =a_{3, k} d_{k} \rho_{k}+\partial_{k} \Omega_{k}^{\prime \prime}, \\
d_{k} a_{3, k} & =\partial_{k} \Omega_{k}^{\prime \prime \prime},
\end{aligned}
$$

where $d_{k}=d / d k$. The flow equations are solved numerically starting at the ultraviolet cutoff scale $\Lambda=1.0 \mathrm{GeV}$ [45]. We eliminate $a_{1}$ by means of the scale independent relation $h=a_{1}(k) \sqrt{2 \rho_{k}}$.

There are four initial conditions for the flow equations, which are fixed at the scale $k=\Lambda$. Within this scheme, the initial value of $a_{0}$ is just a constant shift of thermodynamic potential $\Omega$. We note, however, that such a cutoff at a finite momentum leads to an unphysical behavior of thermodynamic quantities at high temperatures. This problem can be amended by accounting for the $\mu$ - and $T$-dependent contribution of the momenta beyond the cutoff scale [37,49]. Following Ref. [49], we include the high-momentum contribution approximately by using the flow equation for noninteracting massless quarks and gluons,

$$
\begin{aligned}
\partial_{k} \Omega_{k}^{\Lambda}(T, \mu)= & \frac{k^{3}}{12 \pi^{2}}\left\{2\left(N_{c}^{2}-1\right)\left[1+2 n_{B}(k)\right]\right. \\
& \left.-v_{q}\left[1-n_{F}\left(k^{+}\right)-n_{F}\left(k^{-}\right)\right]\right\} .
\end{aligned}
$$

By integrating the flow equation (7) from $k=\infty$ to $k=\Lambda$, we obtain $\Omega^{\Lambda}(T, \mu)$ which is then used as initial condition $a_{0}(\Lambda)$ for the solution of the flow equations (6).

We set $a_{3}(\Lambda)=0$ and fix $\rho_{k=\Lambda}$ and $a_{2}(\Lambda)$ by requiring that in vacuum the pion $m_{\pi}=135 \mathrm{MeV}$ and the sigma 
$m_{\sigma}=640 \mathrm{MeV}$ masses are reproduced. The strength of the Yukawa coupling, $g=3.2$, is fixed by the constituent quark mass, $\quad M_{q}(T=\mu=0)=g \sigma_{k=0}(T=\mu=0)=300 \mathrm{MeV}$ with $\sigma_{k=0}(T=\mu=0)=f_{\pi}=93 \mathrm{MeV}$. The full thermodynamic potential density of the quark-meson model $\Omega(T, \mu)$, which includes thermal and quantum fluctuations of the meson and quark fields, is then obtained from $\Omega(T, \mu)=\lim _{k \rightarrow 0} \Omega_{k}$, where $\Omega_{k}$ is the solution of the flow equation (3) and (6).

By ignoring the mesonic contribution in the flow equation (3), we obtain the effective potential corresponding to the mean-field approximation, with a finite cutoff $\Lambda$. The fermionic vacuum fluctuations, which are included in the mean-field (MF) potential, are necessary to reproduce the second order phase transition at vanishing $\mu$ in the chiral limit $[21,46]$. The vacuum contribution must be renormalized to remove the artificial cutoff dependence [21]. Then, in the meanfield approximation, the thermodynamic potential is given by

$$
\begin{aligned}
\Omega_{\mathrm{MF}}(\langle\sigma\rangle ; T, \mu)= & U(\langle\sigma\rangle, \vec{\pi}=0)-\frac{v_{q}}{16 \pi^{2}} M_{q}^{4} \ln \left(\frac{M_{q}}{M}\right) \\
& -v_{q} T \int \frac{d^{3} p}{(2 \pi)^{3}}\left[\ln \left(1+e^{-\left(E_{q}-\mu\right) / T}\right)\right. \\
& \left.+\ln \left(1+e^{-\left(E_{q}+\mu\right) / T}\right)\right],
\end{aligned}
$$

where $M$ is an arbitrary renormalization scale parameter, $M_{q}=g\langle\sigma\rangle$, and the expectation value $\langle\sigma\rangle$ is determined by the solution of the gap equation $\partial \Omega_{\mathrm{MF}} / \partial\langle\sigma\rangle=0$.

\section{PROBABILITY DISTRIBUTION OF THE NET QUARK NUMBER}

The thermodynamical potentials for the quark-meson model, derived in the previous section, can be used to assess the influence of the underlying chiral phase transition on observables. In the following, we focus on the probability distribution of the net quark number, $P(N)$ and the corresponding cumulants $c_{n}(T, \mu)$.

\section{A. General properties of $\boldsymbol{P}(N)$}

We consider a thermodynamic system described by the grand canonical ensemble at temperature $T$ in a subvolume $V$. We introduce a chemical potential $\mu$ which is used to tune the corresponding average net charge. For the net quark number $N=N_{q}-N_{\bar{q}}$, the probability distribution $P(N)$ to find the net charge $N$ in volume $V$ is given by

$$
P(N ; T, \mu, V)=\frac{Z(T, N, V)}{\mathcal{Z}(T, \mu, V)} e^{\mu N / T},
$$

where $Z(T, N, V)$ is the canonical and $\mathcal{Z}(T, \mu, V)$ the grandcanonical partition function. The normalization of the probability distribution, $\sum_{N=-\infty}^{\infty} P(N)=1$, follows from the fugacity expansion of the grand canonical partition function

$$
\mathcal{Z}(T, \lambda, V)=\sum_{N} \lambda^{N} Z(T, N, V),
$$

where $\lambda=e^{\mu / T}$. Consequently, all essential information on $P(N)$ is contained in the canonical partition function. Since the fugacity expansion is a Laurent series in the complex $\lambda$ plane, with coefficients given by the canonical partition function, the latter can be obtained by performing the contour integral,

$$
Z(T, N, V)=\frac{1}{2 \pi i} \oint_{C} d \lambda \frac{\mathcal{Z}(T, \lambda, V)}{\lambda^{N+1}} .
$$

Thus, to compute the canonical partition function, one needs to know the analytic structure of $\mathcal{Z}(T, \lambda, V)$ in the complex $\lambda$ plane and to choose an appropriate integration contour. ${ }^{1}$ In chiral effective models, the structure of the singularities associated with the chiral phase transition has been discussed in Refs. [13,50]. In the broken phase, $T<T_{c}(\mu)$, there are no singularities on the unit circle $\lambda=e^{i \theta}$ in the range $0 \leqslant \theta<$ $2 \pi$. Consequently, the canonical partition function is obtained from $[51,52]$,

$$
Z(T, N, V)=\frac{1}{2 \pi} \int_{0}^{2 \pi} d \theta e^{-i \theta N} \mathcal{Z}(T, \theta, V),
$$

where $\theta=\mu_{I} / T$ and $\mu_{I}$ is the imaginary chemical potential $\mu=i \mu_{I}$.

The above equation links the grand canonical partition function in a finite volume $V$, at imaginary chemical potential, to the thermodynamics at fixed net charge $N$. At imaginary $\mu$, the QCD partition function exhibits Roberge-Weiss periodicity, $\mathcal{Z}(T, \theta+2 \pi / 3, V)=\mathcal{Z}(T, \theta, V)$ [53]. The Polyakov loop extended effective chiral models reproduce the Roberge-Weiss periodicity [54-56]. In the quark-meson model employed here, the period of the partition function in imaginary chemical potential $\theta$ is $2 \pi$.

In the present work, we follow Ref. [41] and compute the canonical partition function and the corresponding probability distribution using Eq. (12). The thermodynamic potential density $\Omega=-(T / V) \log \mathcal{Z}$ is obtained in the quark-meson model within the FRG approach as well as in the mean-field approximation.

Because of the oscillatory nature of the integrand, a numerical integration of Eq. (12) becomes unreliable for large $|N|$. The numerical quadrature employed here yields accurate results up to values of $|N|$ corresponding to $P(N ; \mu=0) \sim$ $10^{-12}$, independent of the volume, temperature and other parameters. As we show, the achieved precision is sufficient for studying the influence of chiral criticality on the properties of the net quark number probability distribution.

\section{B. Net quark number probability distribution near the chiral phase transition}

At vanishing and moderate values of $\mu$, the quark-meson model in the chiral limit exhibits the second order phase transition, belonging to the $O(4)$ universality class [45]. For a physical pion mass, the chiral symmetry is explicitly broken and the transition is of the crossover type. Nevertheless, remnants of $O(4)$ criticality remain in various observables [5].

\footnotetext{
${ }^{1}$ In a finite system, the grand partition function $\mathcal{Z}$ has Yang-Lee zeros at complex values of $\lambda$. In the thermodynamic limit, the zeros join into cuts.
} 

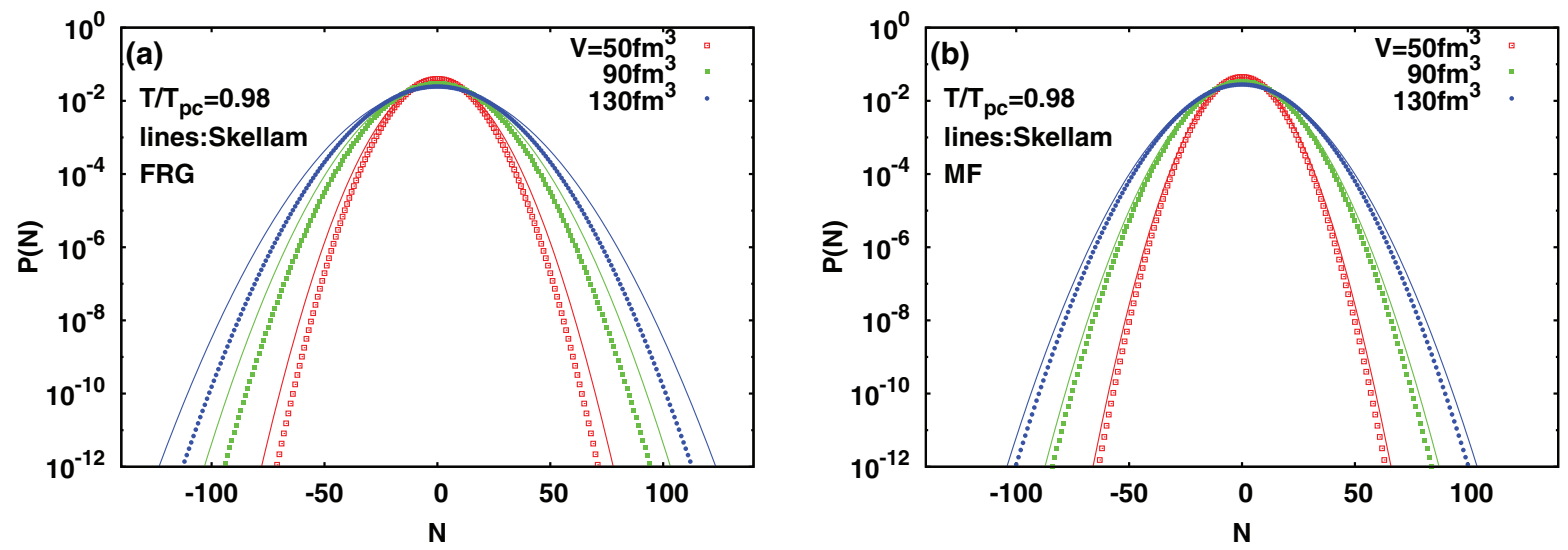

FIG. 1. (Color online) Probability distributions of the net quark number just below the pseudocritical temperature $T_{p c}$ (a) in the FRG approach and (b) in the mean-field (MF) approximation, compared with the corresponding Skellam distributions. The dots are the model results for different volumes while the lines show the Skellam distribution.

Thus, also the probability distribution of the net quark number is expected to exhibit characteristic features reflecting the critical behavior of the underlying $O(4)$ transition. We note, that long range critical correlations can be unfolded only in a sufficiently large sub-volume $V$, and close to the pseudocritical temperature, $T_{p c}$.

To unravel the influence of chiral transition on the probability distribution $P(N)$, one needs to establish a reference distribution, which does not include the effect of critical fluctuations. At low temperatures, $T \ll T_{p c}$, the thermodynamic potential is expected to be well described as a quasi-ideal quark gas with a dynamically generated temperature-dependent mass. Consequently, at fixed $T$ and $V$, the natural reference for $P(N)$ is the probability distribution of an ideal gas of particles and antiparticles, i.e., the Skellam distribution [16]. The Skellam distribution is then determined entirely by the mean number of quarks $b=\left\langle N_{q}\right\rangle$ and antiquarks $\bar{b}=\left\langle N_{\bar{q}}\right\rangle$,

$$
P(N)=\left(\frac{b}{\bar{b}}\right)^{N / 2} I_{N}(2 \sqrt{b \bar{b}}) e^{-(b+\bar{b})}
$$

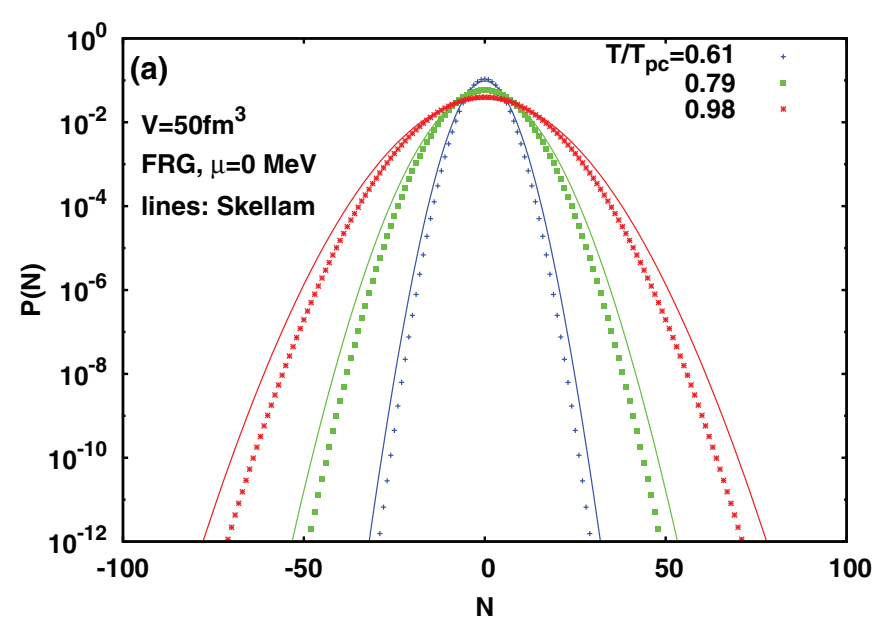

where $I_{N}(x)$ is the modified Bessel function of the first kind. The mean number of quarks $b$ is calculated as for an ideal gas of constituent quarks with a dynamically generated mass

$$
b=\frac{v_{q} V}{2 \pi^{2}} \int_{0}^{\infty} d k k^{2} n_{F}\left(E_{k} ; T, \mu\right),
$$

where $n_{F}$ is the Fermi distribution function and $E_{k}=$ $\sqrt{k^{2}+M_{q}^{2}}$ is the energy of a particle with momentum $k$. The $\bar{b}$ is obtained from Eq. (14) by replacing $\mu \rightarrow-\mu$.

In the MF approximation $M_{q}=g\langle\sigma\rangle$, while in the FRG approach, we use the scale dependent mass $M_{q, k}=g \sigma_{k}$, which for $k \leqslant \Lambda$ is obtained from the flow equation (3). For $\Lambda<k<$ $\infty$ the quarks are assumed to be massless and noninteracting, as discussed above.

Figure 1 shows the probability distributions of the net quark number obtained in the quark-meson model, within the FRG and in the MF approximation at vanishing chemical potential and near the pseudocritical temperature $T_{p c}$. The $T_{p c}$ corresponds to the peak position of the chiral susceptibility, which in the FRG and MF calculations are located at $214 \mathrm{MeV}$ and $190 \mathrm{MeV}$, respectively.

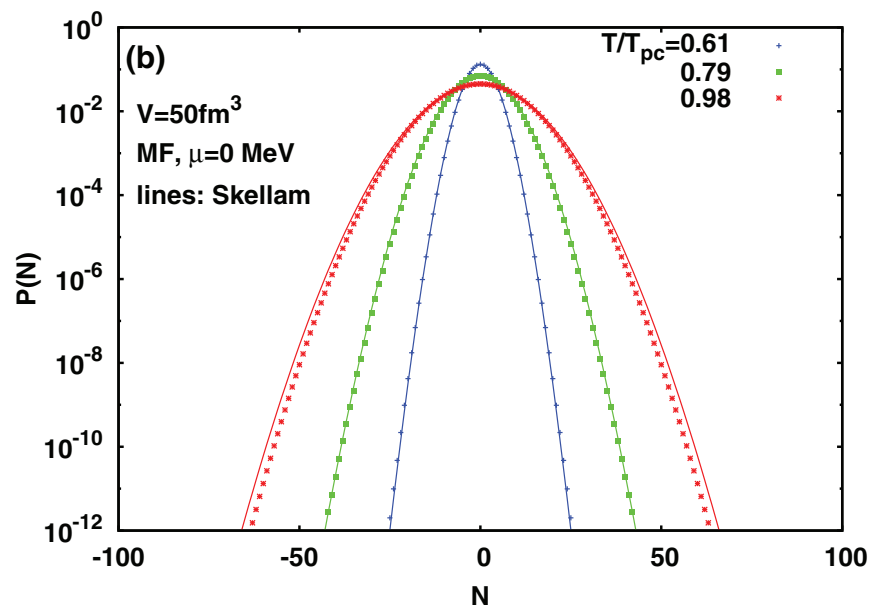

FIG. 2. (Color online) Probability distribution for different temperatures (a) in the FRG approach and (b) in the mean-field (MF) approximation. The dots are the model results while the lines show the corresponding Skellam distribution (see text). 
The probability distributions in Fig. 1 are calculated for different volumes and at fixed temperature. There is a clear change of distributions with the volume as a consequence of a linear dependence of the variance on $V$. The probability distribution also changes rapidly as the temperature is lowered from the pseudocritical point. As shown in Fig. 2, at a given volume, the distributions are narrower at smaller temperatures. This is due to growing mass of quarks, which together with decreasing temperature, imply decreasing mean number of quarks and antiquarks, and consequently the width of the distribution.

The influence of the criticality on the net-quark number probability distribution, and the differences between the MF and the FRG dynamics, are particularly transparent when comparing the results with the noncritical Skellam function.

Figures 1 and 2 show that near $T_{p c}$, both the MF and the FRG distributions are narrower than their Skellam counterparts. Such a reduction of the width of the probability distribution compared to the Skellam distribution was already seen in results obtained previously in the Landau theory of phase transitions when critical fluctuation is included to give negative higher order cumulants [41]. The deviations from the Skellam distribution are stronger when mesonic fluctuations are included, i.e., in the FRG approach. Except for the highest temperature, $P(N)$ in the MF approximation coincides with the Skellam distribution, while in the FRG calculations, the two distributions differ at all temperatures. The increasing difference between the FRG and Skellam distributions, as the temperature approaches $T_{p c}$, reflects the growing influence of mesonic fluctuations leading to the $O(4)$ criticality.

\section{Quantum statistics effects}

The Skellam distribution (13), describes the fluctuations in a gas of non-interacting classical particles with a conserved charge, i.e., particles and antiparticles obeying the Poisson distribution.

In general, effects of quantum statistics should be included if the mass of the particle is smaller than the temperature. The quantum statistics effects were also shown to modify significantly the probability distribution of the electric charge and the resulting cumulants [17,57].

In the FRG approach to the quark-meson model, and at $T / T_{p c}=0.98$ one finds, that $M_{q} / T \simeq 0.6$. Thus, the Boltzmann approximation is clearly not justified near $T_{p c}$.

In order to disentangle the effects of mesonic fluctuations and quantum statistics, we also consider the probability distribution $P(N)$, obtained from Eq. (12), for a free gas of quarks and anti-quarks with the thermodynamic potential density

$$
\begin{aligned}
\Omega_{\mathrm{Fermi}}(T, \theta)= & -\frac{v_{q} T}{2 \pi^{2}} \int_{0}^{\infty} d k k^{2}\left[\ln \left(1+e^{-E_{k} / T+i \theta}\right)\right. \\
& \left.+\ln \left(1+e^{-E_{k} / T-i \theta}\right)\right],
\end{aligned}
$$

and the quark energy $E_{k}$ from Eq. (14).

The effect of Fermi statistics is illustrated in Fig. 3 with the dynamical quark mass obtained in the FRG approach. The probability distribution of the free Fermi gas is seen to be

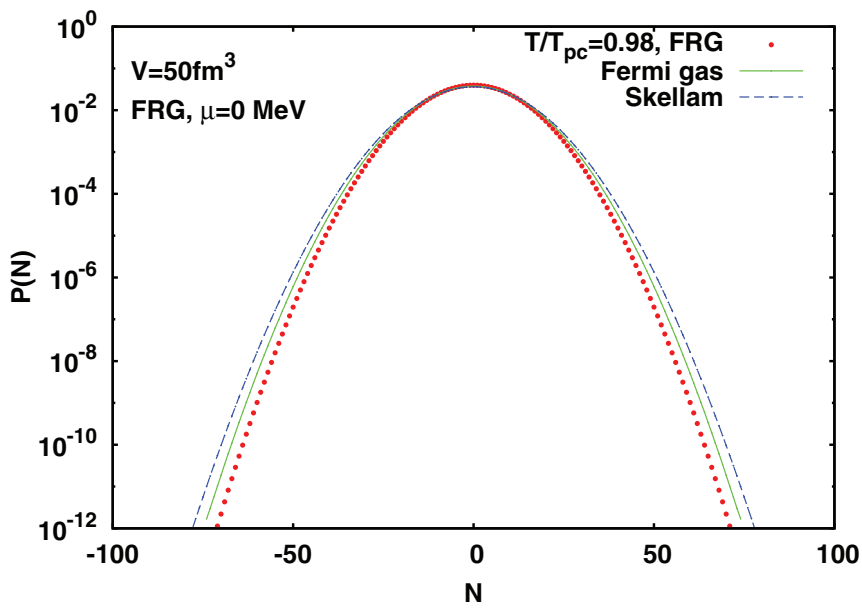

FIG. 3. (Color online) The probability distribution obtained within the FRG approach (red, circles), the Skellam distribution (blue, dashed), and that of a free Fermi gas (green, solid). All distributions correspond to the same average quark and antiquark numbers, $b$ and $\bar{b}$, given by Eq. (14).

narrower than the corresponding Skellam function, but still broader than the $P(N)$ of the quark-meson model in the FRG approach. We identify the residual effect, i.e., the difference between the Fermi gas and the FRG distributions, as being due to the mesonic fluctuations implying the $O(4)$ criticality near the chiral crossover transition.

On the other hand, in the MF approach at $T / T_{p c}=0.98$, the net-quark probability distribution was found to be slightly broader than the corresponding distribution obtained for a free Fermi gas. Therefore, in the MF approach, the apparently narrower distribution than the Skellam, is due to the quantum statistics effects. Thus, there is a clear difference in the properties of the net-quark distributions obtained under MF and the FRG approach. The mesonic fluctuations result in a shrinking of the distribution, whereas the MF critical dynamics results in a broadening of the distribution, relatively to the nonsingular Fermi gas reference, as shown in [41].

\section{Cumulants of the net quark number}

Fluctuations of the net-quark number are quantified by the corresponding cumulants $c_{n}(T, \mu)$, which in turn reflect critical fluctuations. Consequently, cumulants can be used to probe the phase diagram of QCD [5,8-10,16,21-23].

In statistical physics, the cumulants are related to the corresponding susceptibilities,

$$
c_{n}(T, \mu) \equiv \frac{\partial^{n}\left[p(T, \mu) / T^{4}\right]}{\partial(\mu / T)^{n}} .
$$

Thus, given the thermodynamic pressure $p(T, \mu, V)=$ $(T / V) \ln \mathcal{Z}$ in the grand-canonical ensemble, the cumulants $c_{n}(T, \mu)$ can be obtained by taking derivatives of the thermodynamic pressure with respect to the chemical potential.

The cumulants in Eq. (16) can be also obtained from the probability distribution $P(N)$, through the central moments, 


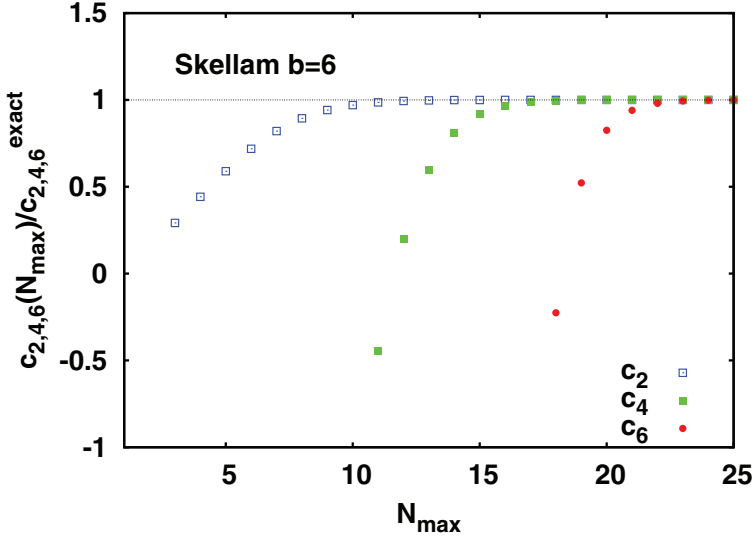

FIG. 4. (Color online) The ratios of the cumulants $c_{n}(n=2,4,6)$ of the net charge obtained from the Skellam distribution with the mean multiplicities $b=\bar{b}=6.0$ using Eqs. (17)-(20) for a given value of $N_{\max }$ to their corresponding exact values.

$\left\langle(\delta N)^{k}\right\rangle=\left\langle(N-\langle N\rangle)^{k}\right\rangle$, where

$$
\left\langle N^{k}\right\rangle=\sum_{N=-N_{\max }}^{N_{\max }} N^{k} P(N),
$$

and $c_{n}$ are given then as polynomials in $\left\langle(\delta N)^{k}\right\rangle$. At vanishing chemical potential, the first three nonvanishing cumulants read

$$
\begin{aligned}
c_{2}= & \frac{\left\langle(\delta N)^{2}\right\rangle}{V T^{3}}, \\
c_{4}= & \frac{\left\langle(\delta N)^{4}\right\rangle-3\left\langle(\delta N)^{2}\right\rangle^{2}}{V T^{3}}, \\
c_{6}= & {\left[\left\langle(\delta N)^{6}\right\rangle-15\left\langle(\delta N)^{4}\right\rangle\left\langle(\delta N)^{2}\right\rangle\right.} \\
& \left.-10\left\langle(\delta N)^{3}\right\rangle^{2}+30\left\langle(\delta N)^{2}\right\rangle^{3}\right] /\left(V T^{3}\right) .
\end{aligned}
$$

In principle, $N_{\max }$ in Eq. (17) is infinite. In practice, however, $N_{\max }$ is always finite. Thus, the cumulants obtained from Eqs. (17)-(20) are, in most cases, approximations to the exact results obtained from Eq. (16).

Figure 4 shows different ratios of cumulants for the Skellam distribution, calculated for fixed mean number of quarks and antiquarks, from Eqs. (17)-(20) for different $N_{\max }$. Clearly, to reproduce the exact results $c_{2 n}=(b+\bar{b}) / V T^{3}$, one needs
$P(N)$ for a sufficiently large $N=N_{\max }$. This value increases with the order of the cumulant, and also depends on the volume, temperature, and the chemical potential.

Figure 5 shows different cumulants obtained in the quarkmeson model within the FRG approach from Eq. (16) and their approximate values computed from Eqs. (17)-(20) as functions of temperature, for several values of $N_{\max }$. For comparison, the cumulants obtained from the corresponding Skellam distribution are also shown in this figure. One notes, that $c_{2}$ and $c_{4}$ of the Skellam and FRG distributions differ, while $c_{6}$ agrees for temperatures well below $T_{p c}$. This behavior can be linked to the $\mu$ dependence of the dynamical quark mass, which at $T \ll T_{p c}$ saturates as the fourth order polynomial in $\mu$, which is not included in the calculations of $c_{n}$ from the Skellam function.

The convergence properties of the cumulants with $N_{\max }$ in the quark-meson model are similar to those found for the Skellam distribution. The value of $N_{\max }$ needed to obtain a good approximation, grows with the order of the cumulant. This reflects the fact, that cumulants of higher order are more sensitive to the tail of the distribution. For the parameter set used in Fig. 5, all cumulants up to the sixth order are well reproduced with $N_{\max } \simeq 90$. This also confirms the consistency of the calculation of $P(N)$ within the quark-meson model.

At $\mu=0$, the second and the fourth order cumulants are not influenced by the critical chiral dynamics, since they remain finite, even in the chiral limit. Thus, their properties are entirely determined by the regular part of the partition function. The temperature dependence of $c_{2}$ and $c_{4}$, seen in Fig. 5, is essentially that of an ideal quark gas, with a modified dispersion relation by $T$ and $\mu$ dependence of a dynamical quark mass.

In contrast, the temperature dependence of $c_{6}$, and in particular its negative values near $T_{p c}$, seen in Fig. 5, are universal. The characteristic shape of $c_{6}$ is generic for the $O(4)$ universality class, owing to the form of the scaling function [5]. The sixth order cumulant obtained from the non-critical Skellam distribution, exhibits a very different behavior.

It is interesting to note, that already for moderate values of $N_{\text {max }}$, the $O(4)$ shape of $c_{6}$ is qualitatively reproduced from the probability distribution. This result is of interest for the eventby-event analysis of heavy ion collisions, where one expects to see the $O(4)$ criticality by reconstructing the higher order
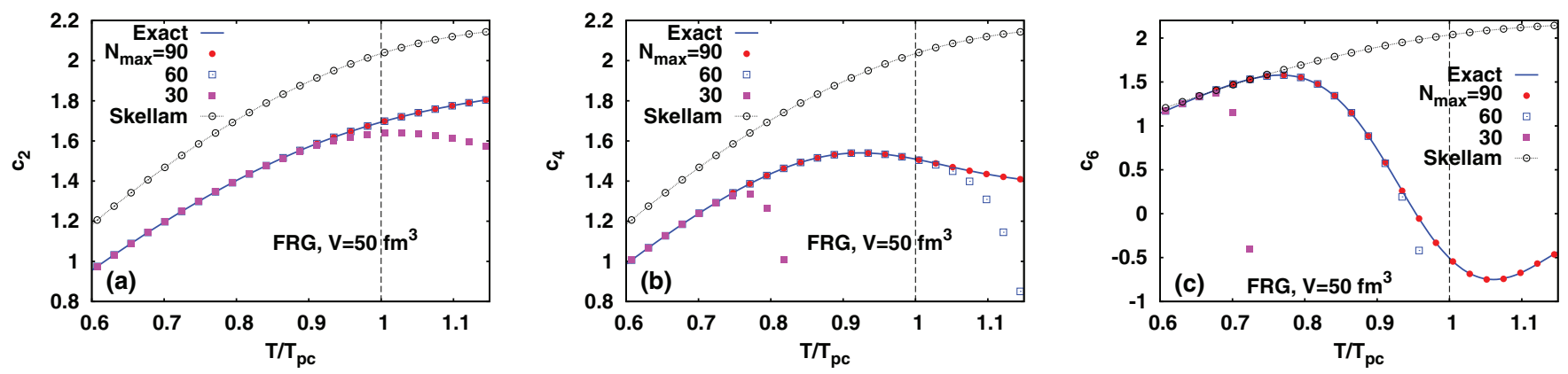

FIG. 5. (Color online) Cumulants $c_{n}(n=2,4,6)$ obtained in the quark-meson model within the FRG approach using Eq. (16) (full line) and Eqs. (17)-(20) (points) for several values of $N_{\max }$. Results for the corresponding Skellam distribution are also indicated by the lines with open circles. 

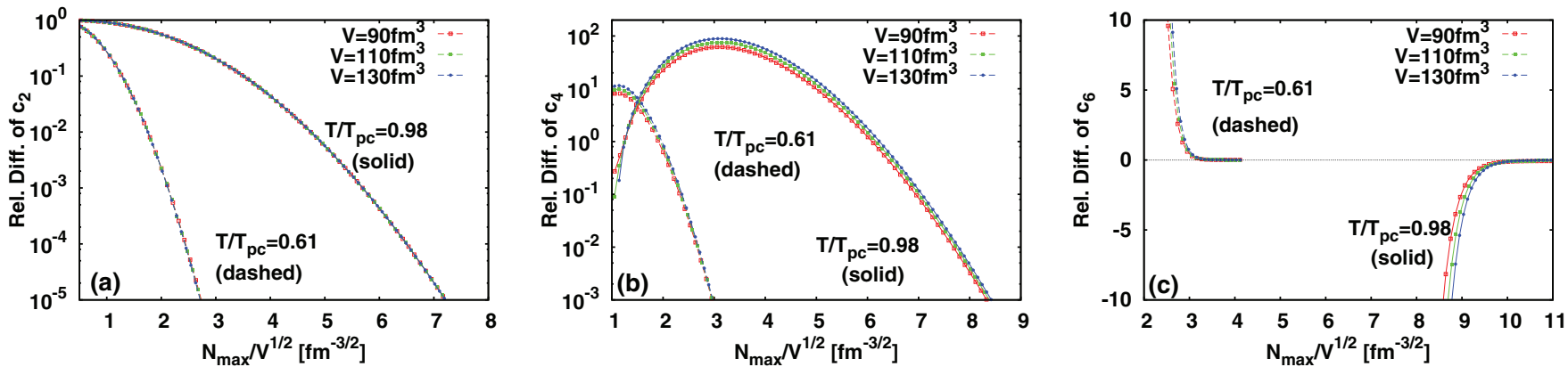

FIG. 6. (Color online) Relative difference between cumulants $c_{n}(n=2,4,6)$ calculated in the quark-meson model within the FRG approach using Eq. (16) and Eqs. (17)-(20) for two temperatures and for several values of the volume parameters $V$ (see text).

moments from the measured net charge probability distribution [24].

The deviations of the approximate cumulants $c_{n}^{A}$, obtained from the probability distribution (17)-(20), from their exact values $c_{n}^{E}$, given by Eq. (16), depend on the volume of the system. The relative deviations of $c_{n}^{A}$ from $c_{n}^{E}$ at fixed $T$ for different $V$, however, obey approximate scaling relations. This is transparent from Fig. 6, showing the relative difference, $R=\left(c_{n}^{E}-c_{n}^{A}\right) / c_{n}^{E}$ for $n=2,4,6$, as a function of $N_{\max } / \sqrt{V}$. For $n=2$ there is a clear scaling for all $N_{\max }$. For higher order cumulants, the approximate scaling becomes better for larger values of $N_{\max } / \sqrt{V}$.

Since the cumulants of the net quark number are linked to the corresponding probability distribution through Eqs. (17)-(20), the approximate scaling of the cumulants, seen in Fig. 6, should be also reflected in the net quark number probability distributions. Indeed, Fig. 7 shows, that for fixed $T$, the $\sqrt{V T^{3}} P(N)$ scales approximately with $N / \sqrt{V T^{3}}$. This approximate scaling is valid for $P(N)$, calculated in the quarkmeson model within the FRG and MF approach, as well as, for the corresponding Skellam distributions. Figure 7 indicates,

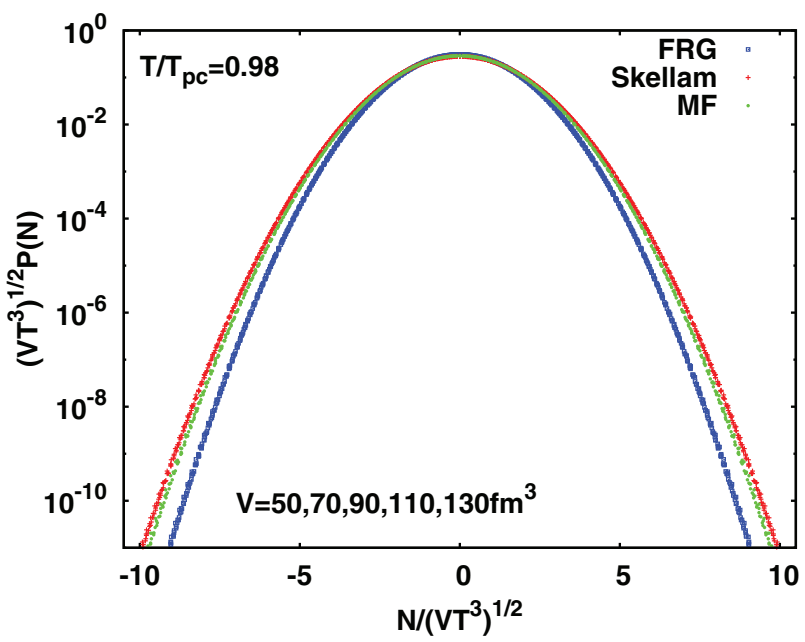

FIG. 7. (Color online) Volume scaling of the net quark number probability distribution at fixed $T / T_{p c}$ in the quark-meson model within the MF, the FRG approach, and for the corresponding Skellam distribution. that the shape of the distribution reflects the underlying criticality in a system which is governed by the critical exponents. The probability distribution in the MF case is broader than in the FRG approach, owing to differences caused by the mesonic fluctuations. As they smoothen the transition, the resultant dynamical quark mass is heavier than the MF case at the same $T / T_{p c}$ [37], leading to narrower distribution.

\section{CONCLUDING REMARKS}

We have studied the properties of the probability distribution $P(N)$ of the net quark number in the presence of the critical chiral dynamics governed by the $O(4)$ universality class in the quark-meson model. The computations of $P(N)$ have been done within the Functional Renormalization Group (FRG) approach, which preserves the $O(4)$ scaling of relevant physical observables.

The main objectives of this paper was to study the influence of the underlying chiral phase transition on the net quark probability distribution, for a physical value of the pion mass.

We have shown, by comparing the FRG and the mean-field (MF) results, that the shape of the distribution reflects the criticality in a system. The FRG distribution is narrower than the one obtained in the MF approximation. This is mainly due to differences in the values of dynamically generated quark mass. Effects of the expected $O(4)$ criticality appear in the tail of the distribution and imply characteristic shapes of the higher order cumulants.

Near the chiral crossover transition, the probability distribution of the net quark number was also shown to be narrower than the Skellam function, which corresponds to a classical quasiparticle gas and is used as a reference for the noncritical distribution. The narrowing of the probability distribution is mainly due to mesonic fluctuations. However, owing to dropping quark mass near the chiral transition also quantum statistics play a role, although a subleading one.

The observed shrinking of $P(N)$ relative to the Skellam distribution is also expected in the $O(2)$ universality class. This is because, the scaling functions in the $O(4)$ and $O(2)$ universality classes are very similar [14] and exhibit negative values of the specific heat critical exponents $\alpha$. However, since $\alpha$ in $O(2)$ is larger than in $O(4)$ universality class, one expects quantitative differences in the properties of $P(N)$. 
We have found an approximate scaling of the probability distribution and of the net charge cumulants with the volume of the system. This implies, that the observed properties of $P(N)$ near the chiral transition are volume independent, and are due to mesonic fluctuations implying $O(4)$ criticality near the chiral crossover transition.

These results are of importance in heavy ion collisions, where by measuring the net baryon number probability distribution and related moments, one expects to experimentally probe the QCD phase boundary. The phenomenological implications of our results will be presented elsewhere [58].

\section{ACKNOWLEDGMENTS}

The authors acknowledge stimulating discussions with P. Braun-Munzinger, F. Karsch, and $\mathrm{Nu} \mathrm{Xu}$. K.M. was supported by Yukawa International Program for Quark-Hadron Sciences at Kyoto University and by the Grant-in-Aid for Scientific Research from JSPS No. 24540271. B.F. is supported in part by the Extreme Matter Institute EMMI. K.R. acknowledges partial support of the Polish Ministry of National Education (MEN). The research of V.S. is supported under Contract No. DE-AC02-98CH10886 with the U.S. Department of Energy.
[1] B. Friman, C. Höhne, J. Knoll, S. Leupold, J. Randrup, R. Rapp, and P. Senger, Lect. Note Phys. 814, 1 (2011).

[2] K. Fukushima and T. Hatsuda, Rep. Prog. Phys. 74, 014001 (2011).

[3] M. Asakawa and K. Yazaki, Nucl. Phys. A 504, 668 (1989).

[4] Z. Fodor and S. D. Katz, J. High Energy Phys. 03 (2002) 014; C. Schmidt, C. R. Allton, S. Ejiri, S. J. Hands, O. Kaczmarek, F. Karsch, and E. Laermann, Nucl. Phys. Proc. Suppl. 119, 517 (2003); R. V. Gavai, Nucl. Phys. A 862, 104 (2011).

[5] F. Karsch and K. Redlich, Phys. Lett. B 695, 136 (2011).

[6] Y. Hatta and M. A. Stephanov, Phys. Rev. Lett. 91, 102003 (2003).

[7] M. Stephanov, K. Rajagopal, and E. Shuryak, Phys. Rev. Lett. 81, 4816 (1998).

[8] S. Ejiri, F. Karsch, and K. Redlich, Phys. Lett. B 633, 275 (2006).

[9] M. A. Stephanov, Phys. Rev. Lett. 102, 032301 (2009).

[10] M. A. Stephanov, Phys. Rev. Lett. 107, 052301 (2011).

[11] R. D. Pisarski and F. Wilczek, Phys. Rev. D 29, 338 (1984).

[12] Y. Aoki, G. Endrödi, Z. Fodor, S. D. Katz, and K. K. Szabó, Nature 443, 675 (2006).

[13] V. Skokov, K. Morita, and B. Friman, Phys. Rev. D 83, 071502(R) (2011); B. Friman, Acta. Phys. Pol. B (Proc Suppl.) 5, 707 (2012).

[14] S. Ejiri, F. Karsch, E. Laermann, C. Miao, S. Mukherjee, P. Petreczky, C. Schmidt, W. Soeldner, and W. Unger, Phys. Rev. D 80, 094505 (2009).

[15] A. Bazavov, T. Bhattacharya, M. Cheng, C. DeTar, H. T. Ding, S. Gottlieb, R. Gupta, P. Hegde et al., Phys. Rev. D 85, 054503 (2012).

[16] P. Braun-Munzinger, B. Friman, F. Karsch, K. Redlich, and V. Skokov, Phys. Rev. C 84, 064911 (2011).

[17] P. Braun-Munzinger, B. Friman, F. Karsch, K. Redlich, and V. Skokov, Nucl. Phys. A 880, 48 (2012).

[18] O. Kaczmarek, F. Karsch, E. Laermann, C. Miao, S. Mukherjee, P. Petreczky, C. Schmidt, W. Soeldner, and W. Unger, Phys. Rev. D 83, 014504 (2011).

[19] A. Bazavov et al. (HotQCD Collaboration), Phys. Rev. D 86, 034509 (2012).

[20] A. Bazavov, H. T. Ding, P. Hegde, O. Kaczmarek, F. Karsch, E. Laermann, S. Mukherjee, P. Petreczky et al., Phys. Rev. Lett. 109, 192302 (2012).

[21] V. Skokov, B. Friman, E. Nakano, K. Redlich, and B.-J. Schaefer, Phys. Rev. D 82, 034029 (2010).

[22] B. Friman, F. Karsch, K. Redlich, and V. Skokov, Eur. Phys. J. C 71, 1694 (2011).
[23] V. Skokov, B. Friman, and K. Redlich, Phys. Rev. C 83, 054904 (2011).

[24] M. M. Aggarwal et al. (STAR Collaboration), Phys. Rev. Lett. 105, 022302 (2010).

[25] X. Luo (STAR Collaboration), Nucl. Phys. A 904-905, 911c (2013); L. Chen (STAR Collaboration), ibid. 904-905, 471c (2013).

[26] M. Kitazawa and M. Asakawa, Phys. Rev. C 85, 021901(R) (2012).

[27] M. Kitazawa and M. Asakawa, Phys. Rev. C 86, 024904 (2012); 86, 069902(E) (2012).

[28] A. Bzdak, V. Koch, and V. Skokov, Phys. Rev. C 87, 014901 (2013).

[29] A. Bzdak and V. Koch, Phys. Rev. C 86, 044904 (2012).

[30] C. R. Allton, M. Döring, S. Ejiri, S. J. Hands, O. Kaczmarek, F. Karsch, E. Laermann, and K. Redlich, Phys. Rev. D 71, 054508 (2005).

[31] S. Borsanyi, Z. Fodor, S. D. Katz, S. Krieg, C. Ratti, and K. Szabo (Wuppertal-Budapest Collaboration), J. High Energy Phys. 01 (2012) 138.

[32] M. Cheng, P. Hegde, C. Jung, F. Karsch, O. Kaczmarek, E. Laermann, R. D. Mawhinney, C. Miao, P. Petreczky, C. Schmidt, and W. Soeldner, Phys. Rev. D 79, 074505 (2009).

[33] K. Fukushima, Phys. Lett. B 591, 277 (2004).

[34] C. Sasaki, B. Friman, and K. Redlich, Phys. Rev. D 75, 054026 (2007).

[35] C. Sasaki, B. Friman, and K. Redlich, Phys. Rev. D 75, 074013 (2007).

[36] B. Stokic, B. Friman, and K. Redlich, Phys. Lett. B 673, 192 (2009).

[37] V. Skokov, B. Stokic, B. Friman, and K. Redlich, Phys. Rev. C 82, 015206 (2010).

[38] M. Asakawa, S. Ejiri, and M. Kitazawa, Phys. Rev. Lett. 103, 262301 (2009).

[39] T. K. Herbst, J. M. Pawlowski, and B. J. Schaefer, Phys. Lett. B 696, 58 (2011).

[40] B.-J. Schaefer and M. Wagner, Phys. Rev. D 85, 034027 (2012); M. Wagner, A. Walther, and B.-J. Schaefer, Comp. Phys. Comm. 181, 756 (2010).

[41] K. Morita, V. Skokov, B. Friman, and K. Redlich, arXiv:1211.4703 [hep-ph].

[42] C. Wetterich, Phys. Lett. B 301, 90 (1993).

[43] J. Berges, N. Tetradis, and C. Wetterich, Phys. Rep. 363, 223 (2002).

[44] B. Delamotte, cond-mat/0702365. 
[45] B. Stokić, B. Friman, and K. Redlich, Eur. Phys. J. C 67, 425 (2010).

[46] E. Nakano, B. J. Schaefer, B. Stokic, B. Friman, and K. Redlich, Phys. Lett. B 682, 401 (2010).

[47] B.-J. Schaefer and J. Wambach, Phys. Rev. D 75, 085015 (2007).

[48] K. Kamikado, T. Kunihiro, K. Morita, and A. Ohnishi, Prog. Theor. Exp. Phys. 053D01 (2013).

[49] J. Braun, H.-J. Pirner, and K. Schwenzer, Phys. Rev. D 70, 085016 (2004).

[50] M. A. Stephanov, Phys. Rev. D 73, 094508 (2006).

[51] P. Braun-Munzinger, K. Redlich, and J. Stachel, in Quark-Gluon Plasma 3, edited by R. C. Hwa and X. N. Wang (World Scientific, Singapore, 2004).
[52] R. Hagedorn and K. Redlich, Z. Phys. C 27, 541 (1985).

[53] A. Roberge and N. Weiss, Nucl. Phys. B 275, 734 (1986).

[54] Y. Sakai, K. Kashiwa, H. Kouno, and M. Yahiro, Phys. Rev. D 77, 051901(R) (2008).

[55] K. Morita, V. Skokov, B. Friman, and K. Redlich, Phys. Rev. D 84, 076009 (2011).

[56] K. Morita, V. Skokov, B. Friman, and K. Redlich, Phys. Rev. D 84, 074020 (2011).

[57] V. Skokov, B. Friman, and K. Redlich, Phys. Lett. B 708, 179 (2012).

[58] K. Morita, talk given at the 4th Asian Triangle Heavy Ion Conference (ATHIC2012), November 16, 2012, Pusan, Korea; K. Morita, B. Friman, K. Redlich, and V. Skokov, in preparation. 